\title{
Satisfação do usuário da Atenção Primária no Distrito Federal: a importância do acesso oportuno e da visita domiciliar
}

\author{
Primary Health Care users' satisfaction in the Federal District: the \\ importance of timely access and home visits
}

\author{
Denise de Lima Costa Furlanetto (https://orcid.org/0000-0002-5703-7520) ${ }^{1}$ \\ Adriano de Almeida de Lima (https://orcid.org/0000-0002-4125-6980) ${ }^{2}$ \\ Claudia Mara Pedrosa (https://orcid.org/0000-0001-9253-3928) ${ }^{1}$ \\ Thatianny Tanferri de Brito Paranaguá (https://orcid.org/0000-0003-0562-8975) ${ }^{3}$ \\ Mayra Fernandes Xavier (https://orcid.org/0000-0002-1885-9513) ${ }^{1}$ \\ Amanda Kellen Pereira da Silva (https://orcid.org/0000-0001-6612-6130) ${ }^{1}$ \\ Viviane Belini Rodrigues (https://orcid.org/0000-0002-2389-820X) ${ }^{4}$ \\ Leonor Maria Pacheco Santos (https://orcid.org/0000-0002-6739-6260) ${ }^{1}$
}

${ }^{1}$ Programa de PósGraduação em Saúde Coletiva, Departamento de Saúde Coletiva, Faculdade de Ciências da Saúde, Universidade de Brasília (UnB). Campus Universitário Darcy Ribeiro s/n, Asa Norte. 70910900 Brasília DF Brasil. denisefurlanetto@unb.br ${ }^{2}$ Departamento de Odontologia, Faculdade de Ciências da Saúde, UnB.

Brasília DF Brasil. ${ }^{3}$ Departamento de Enfermagem, Faculdade de Ciências da Saúde, UnB. Brasília DF Brasil.

${ }^{4}$ Departamento de Nutrição, Faculdade de Ciências da Saúde, UnB. Brasília DF Brasil.

\begin{abstract}
The aim of this cross-sectional study was to analyze user satisfaction in relation to Primary Health Care services in the Federal District and its associated factors. A total of 4,476 users of the 62 existing Family Health Strategy Teams from the Eastern Health Region were included; a pre-tested questionnaire with open and closed questions was applied. As for the "General Satisfaction Level" $54.9 \%$ of users were Satisfied and $23.0 \%$ Very Satisfied. For this outcome there was less satisfaction in cases that the user didn't receive the care he sought for (OR $12.1 p=0.01)$; did not receive a home visit (OR $1.7 p=0.01)$; is female (OR $1.2 p=0.01)$ and declared to be nonwhite (OR $0.77 p=0.021)$. The "Chance to recommend the Health Unit" was: High (43.4\%) and Very High (17.7\%). Similarly, the lower chance to recommend was associated with no care received (OR $5.1 p=0.01)$ and absence of home visit (OR $1.5 p=0.01$ ); there was no association with sociodemographic variables. Evidence showed that health services are satisfactory for most users. The study provides evidence regarding the importance of having the complete Family Health Strategy team and of expanding home visit.
\end{abstract}

Key words Patient Satisfaction, Health services, Home visit, Primary Health Care
Resumo Trata-se de estudo transversal, com o objetivo de analisar a satisfação de usuários em relação ao atendimento em unidades de Atenção Primária à Saúde do Distrito Federal e fatores associados. Foram incluídos 4.476 usuários das 62 equipes da ESF existentes na Região de Saúde Leste; utilizou-se questionário testado previamente com perguntas abertas e fechadas. Quanto ao nível geral de satisfação, encontrou-se 54,9\% dos usuários Satisfeitos e 23,0\% Muito Satisfeitos. Para este desfecho Nivel Geral de Satisfação houve menor satisfação nos casos que o usuário não conseguiu ser atendido (OR 12,1 $p=0,01$ ); não recebeu visita domiciliar (OR 1,7 $p=0,01)$; é do sexo feminino (OR 1,2 $p=0,01)$ e declarou cor/etnia não branco (OR 0,77 $p=0,021)$. Já a "Chance do Usuário Recomendar a UBS” foi: Alta $(43,4 \%)$ e Muito Alta (17,7\%). De modo similar, a menor chance de recomendar foi associada a casos de não atendimento (OR 5,1 p=0,01) e ausência de visita domiciliar (OR 1,5 p=0,01); não houve associação com variáveis sociodemográficas. Evidencia-se a percepção que os serviços são satisfatórios para a maioria dos usuários. O estudo também trouxe evidências para a importância de contar com a equipe ESF completa e de expandir a visita domiciliar.

Palavras-chave Satisfação do Paciente, Serviços de saúde, Visita domiciliar, Atenção Primária à Saúde 


\section{Introdução}

No contexto do planejamento e gestão dos serviços de saúde, a avaliação tem sido ferramenta fundamental, especialmente no campo da Saúde Pública, por direcionar decisões mais assertivas e favorecer o controle técnico e social dos serviços prestados à sociedade ${ }^{1,2}$.

$\mathrm{O}$ uso de metodologias participativas tem ganhado destaque nesse cenário por ampliar o escopo das avaliações e, nessa perspectiva, ressaltam-se, como necessidades inerentes ao processo avaliativo, o engajamento dos atores envolvidos na governança regional, compreensão de que a avaliação não é imparcial e objetiva e que ações de melhoria da atenção dependem do envolvimento ativo do cidadão no processo de produção da saúde ${ }^{3,4}$. A satisfação do usuário, nesse sentido, emerge como um componente essencial para avaliar a qualidade dos serviços de saúde, constatado em literatura nacional e internacional ${ }^{5}$, por expressar a capacidade dos serviços em atenderem necessidades e expectativas da população.

A satisfação dos usuários como componente de avaliações dos serviços de saúde surgiu inicialmente nos Estados Unidos e na Europa, entre 1960 e 1970, com a finalidade de analisar aspectos técnicos e estruturais da qualidade da atenção à saúde e adesão ao tratamento. No Brasil, essa inserção aconteceu em meados dos anos de 1990, subsidiada pela necessidade de fortalecer o controle social do Sistema Único de Saúde (SUS) e da participação da comunidade nos processos de planejamento e avaliação, a partir do movimento de corresponsabilização pelo cuidado ${ }^{5-7}$. Iniciativas sobre o cuidado centrado no usuário têm se tornado regular nas políticas, com propostas internacionais, como a inclusão do foco no paciente como uma dimensão da qualidade do cuidado, pelo Institute of $\mathrm{Health}^{8}$. A partir do avanço de iniciativas anteriores, atualmente no Reino Unido o cuidado centrado na pessoa é considerado fundamental nas políticas, com a obrigação legal de envolver os pacientes no processo de cuidado?. No Brasil, esforços nesse sentido têm ocorrido, a exemplo da Política Nacional de Humanização ${ }^{10}$.

Avaliar a satisfação dos usuários oportuniza a construção e apropriação de um conhecimento que favorece a promoção de mudanças organizacionais que tenham significado para os envolvi$\operatorname{dos}^{4,11}$, especialmente para os usuários. Esse movimento contribui para aperfeiçoar os processos de organização dos serviços de saúde, as ações de planejamento em saúde, a tomada de decisão em nível de gestão, assim como influencia na ressignificação de políticas públicas de saúde ${ }^{5}$.
Compreender a satisfação dos usuários como uma dimensão do processo de avaliação, planejamento e gestão do serviço de saúde permite a identificação de espaços e estratégias focados em problemas reais vivenciados pelos usuários ${ }^{4}$, oportunizando mudanças nos processos de trabalho que sejam mais específicas à realidade organizacional e da população.

Num movimento em que se aspira prestar cuidados centrados no paciente, é essencial compreender a qualidade do serviço do ponto de vista do usuário. À medida que estudos incorporam a satisfação do usuário como uma dimensão da qualidade da atenção ${ }^{12}$, fica explicitada a relevância de considerar elementos não técnicos da avaliação. Starfield ${ }^{13}$ afirma que a satisfação de usuários, apesar de não ser diretamente uma medida de qualidade assistencial, é uma medida indireta de qualidade, pois a satisfação pode influenciar a busca pelos serviços e melhoria na saúde das pessoas. Estudos sobre satisfação dos usuários e fatores associados, como humanização do cuidado e integralidade ${ }^{5}$, aspectos médicos e aspectos não relacionados ao cuidado direto ${ }^{12} \mathrm{e}$ dimensões não médicas do atendimento ${ }^{14}$ obtiveram em geral resultados positivos em relação à satisfação dos usuários, com especificidades inerentes a cada fator em questão.

Frente ao exposto, e considerando a importância de envolver a percepção dos usuários nos processos de avaliação dos serviços de saúde, o presente estudo tem como objetivo analisar fatores associados à satisfação dos usuários com ênfase em questões relacionadas ao atendimento oferecido em unidades na Atenção Primária à Saúde, tendo como contexto de análise a Região Leste do Distrito Federal.

\section{Método}

Trata-se de estudo transversal realizado de agosto de 2018 a fevereiro de 2019. Foram incluídos na amostra usuários de todos os serviços de Atenção Primária à Saúde (APS) da Região Leste do Distrito Federal, nas Regiões Administrativas XIV São Sebastião, VII - Paranoá e XXVIII - Itapoã, com população de aproximadamente 216.000 habitantes ${ }^{15}$.

O tamanho da amostra foi calculado com base na prevalência de $90,6 \%$ de avaliação da equipe de enfermagem como atenciosa/respeitosa no estudo de Hollanda et al. ${ }^{14}$, com erro admissível de 1,2 pontos percentuais e $95 \%$ de nível de Confiança; tamanho mínimo da amostra resultou em 4.450 usuários. A unidade amostral foi 
o usuário e a seleção ocorreu entre os usuários de todas as 62 Equipes de Saúde da Família (ESF) existentes nestes territórios.

Para analisar a satisfação dos usuários foram aplicados questionários elaborados segundo a metodologia proposta por Pasquali ${ }^{16}$ para a construção de testes psicológicos. O questionário constava de um bloco para caracterização sociodemográfica dos usuários e dois blocos referentes à satisfação dos usuários e responsividade das UBS. A avaliação da satisfação foi medida através de perguntas indiretas e diretas, utilizando-se escalas do tipo Likert de 5 fases.

O questionário foi validado inicialmente por análise teórica dos itens realizada por especialistas e análise semântica junto a um grupo de usuários de UBS. Após a aplicação, as perguntas de satisfação indireta e responsividade passaram pela análise fatorial em cada um dos blocos de perguntas. As perguntas que apresentaram carga fatorial menor que 0,30 foram excluídas. Depois foi calculada a fidedignidade de cada fator, encontrando-se valores a partir de 0,73. Duas perguntas foram elaboradas para captar a satisfação direta e foram usadas como parâmetro de comparação entre as perguntas validadas e uma medida padrão. A validação do questionário foi objeto de uma dissertação de mestrado.

No presente estudo foram utilizadas essas perguntas de satisfação direta como variáveis de desfechos. As perguntas utilizadas foram: 1) Qual é o seu nível geral de satisfação com o atendimento que recebeu na Unidade Básica de Saúde hoje? e 2) Baseado na sua experiência de hoje, qual é a chance de você recomendar essa Unidade Básica de Saúde a amigos e familiares?

Os critérios de inclusão foram: usuário que buscou qualquer tipo de atendimento na UBS, adulto/idoso (maior ou igual a 18 anos) em condições de responder; adulto/idoso sem condições clínicas de responder (surdez, mudez, senilidade, AVC, deficiência mental/cognitiva) que estivesse com um acompanhante adulto; menor de idade com acompanhante adulto. No caso de usuário sem condições de responder ou menor de idade o acompanhante respondeu ao questionário. Os questionários foram instalados em tablets e entrevistadores previamente treinados aplicaram os questionários em todos os turnos e qualquer horário.

Os dados foram analisados com o software Statistical Package for the Social Sciences, versão 23 (SPSS 23, Chicago, EUA). Foi aplicado o Teste do Qui-quadrado de Independência para determinar se as variáveis sociodemográficas (Sexo, Escolaridade e Cor/Etnia) e de atendimento
(Conseguir realizar o atendimento, Motivo principal da visita à UBS e Visita Domiciliar) são independentes ou associadas com as duas variáveis de desfecho: Nível Geral de Satisfação; e Chance do Usuário Recomendar a UBS. Para medir a magnitude da associação foi utilizado o Teste $\mathrm{V}$ de Cramer.

Para melhorar a sensibilidade e a interpretação do Teste do Qui-quadrado de Independência foram calculados residuais (diferença entre a frequência esperada e a frequência observada) padronizados ajustados, que podem ser positivos (indicando que há mais frequências observadas do que as esperadas) ou negativos (indicando que há menos frequências observadas do que as esperadas). Quanto maior o valor absoluto do resíduo padronizado ajustado, independente do sinal, maior a contribuição considerada para o valor do qui-quadrado e maior a evidência a favor da associação. Foi considerado que os resíduos padronizados ajustados maiores que 3 (erros padrão) produziram desvio significante a favor da associação.

Para as variáveis contínuas Idade, Renda Familiar Mensal e Número de Pessoas que Dependem da Renda foi aplicado o Teste de Correlação de Spearman. As variáveis que mostraram correlação com nível de significância $\geq 90 \%$ foram incluídas em um modelo de Regressão Logística Ordinal. Todas as variáveis testadas apresentaram nível de resposta satisfatório, portanto, os dados perdidos não precisaram de tratamentos ou substituições.

O projeto foi submetido ao Comitê de Ética da Fundação Oswaldo Cruz - Brasília e da Fundação de Ensino e Pesquisa em Ciências da Saúde (FEPECS-DF). Após a explicação dos objetivos do estudo e da assinatura de Termo de Consentimento Livre e Esclarecido, os usuários que voluntariamente aceitaram participar do estudo responderam às perguntas dos questionários. A pesquisa teve boa aceitação e a proporção de recusas foi menor que $5 \%$.

\section{Resultados}

Dos 4.560 questionários preenchidos $84(1,8 \%)$ foram eliminados por não atenderem aos critérios de inclusão da pesquisa, sendo 20 dos entrevistados menores de 18 anos que estavam sem acompanhante adulto; 52 acompanhantes que responderam no lugar de adulto/idoso que tinha condições de responder; 12 pelo fato do acompanhante não declarar a idade do acompanhado. $\mathrm{O}$ total de questionários válidos foi de 4.476, superior ao mínimo calculado (4.450). 
Os dados sociodemográficos dos usuários que responderam aos questionários e o tipo de atendimento que procuraram nos serviços de Atenção Primária à Saúde estão descritos na Tabela 1. Do total de entrevistados, a maioria $(77,4 \%)$ é do sexo feminino. Em relação à cor/etnia, a maioria se declarou parda $(60,0 \%)$, seguida da cor negra $(18,7 \%)$. Em relação à escolaridade predominaram participantes que cursaram o ensino médio $(48,7 \%)$, seguido de $35,3 \%$ com ensino fundamental. A amostra consistiu em $20,1 \%$ de jovens adultos, $44,2 \%$ adultos, $21,2 \%$ de adultos na meia idade e $14,6 \%$ de idosos; a idade média dos usuários foi de 39,7 anos (DP 15,9) e a Media-

Tabela 1. Caracterização sociodemográfica e tipo de atendimento nos serviços de Atenção Primária à Saúde dos usuários incluídos no estudo. Distrito Federal, 2018-2019.

\begin{tabular}{lrr}
\hline \begin{tabular}{l} 
Característica do usuário e/ou tipo \\
\multicolumn{1}{c}{ de atendimento }
\end{tabular} & N & \multicolumn{1}{c}{$\%$} \\
\hline Sexo & & \\
Mulher & 3.465 & 77,4 \\
Homem & 1.006 & 22,5 \\
Outro & 5 & 0,1 \\
Total & 4.476 & 100,0 \\
Cor/Etnia & & \\
Amarela & 163 & 3,6 \\
Branca & 755 & 16,9 \\
Indígena & 35 & 0,8 \\
Parda & 2.685 & 60,0 \\
Preta & 838 & 18,7 \\
Total & 4.476 & 100,0 \\
Escolaridade & & \\
Sem Escolaridade & 251 & 5,6 \\
Ensino Fundamental & 1.578 & 35,3 \\
Ensino Médio & 2.181 & 48,7 \\
Ensino Superior & 466 & 10,4 \\
Total & 4.476 & 100,0 \\
Faixa Etária & & \\
18 a 24 anos & 898 & 20,1 \\
25 a 44 anos & 1977 & 44,2 \\
45 a 59 & 948 & 21,2 \\
Total & 653 & 14,6 \\
Renda Familiar & 4.476 & 100,0 \\
Até 01 salário mínimo & & \\
De 01 a 03 salários mínimos & 973 & 26,9 \\
De 03 a 05 salários mínimos & 2166 & 59,8 \\
Mais de 05 salários mínimos & 362 & 10,0 \\
Total & 122 & 3,4 \\
& & 100,0 \\
\hline
\end{tabular}

na 37 anos. Em relação à Renda Mensal Familiar o valor médio dos 3.623 respondentes foi de $\mathrm{R} \$$ $1.729,00$ (DP R \$ 1.418,00; Mediana R \$ 1.300,00 e Moda R $\$ 1.000,00)$. O número médio de pessoas que dependem da renda familiar foi de 3,5 (DP 1,6; Mediana 3 e Moda 3). A maioria dos entrevistados, 59,8\% tem Renda Familiar Mensal (RFM) entre 1 e 3 salários mínimos e $26,9 \%$ até um salário mínimo. Os dados confirmam o fato que os territórios estudados estão entre as Regiões Administrativas (RA) de menor Renda Familiar Mensal do DF, sendo que Itapoã exibe a $4^{\text {a }}$ menor RFM (PDAD 2015) ${ }^{15}$.

Quanto ao atendimento dos usuários nos serviços incluídos no estudo, $81,7 \%$ dos respondentes relataram ter conseguido realizar o atendimento para o qual procuraram a Unidade de Saúde. Os motivos de consulta que prevaleceram, segundo os relatos, foram: consulta agendada; retirada de medicamento e consulta sem agendamento prévio. A Tabela 1 apresenta, também, a

Tabela 1. Caracterização sociodemográfica e tipo de atendimento nos serviços de Atenção Primária à Saúde dos usuários incluídos no estudo. Distrito Federal, 2018-2019.

\begin{tabular}{lrr}
\hline $\begin{array}{c}\text { Característica do usuário e/ou tipo } \\
\text { de atendimento }\end{array}$ & N & $\%$ \\
\hline $\begin{array}{l}\text { Conseguiu realizar o atendimento para o qual } \\
\text { procurou a UBS? }\end{array}$ & \\
$\quad$ Não & 819 & 18,3 \\
Sim & 3.646 & 81,7 \\
Total & 4.465 & 100,0 \\
Motivo principal da visita à UBS & & \\
Agendamento de consulta & 526 & 11,8 \\
Consulta agendada & 1.102 & 24,6 \\
Consulta sem agendamento prévio & 776 & 17,3 \\
Curativo & 84 & 1,9 \\
Grupo Educativo & 34 & 0,8 \\
Nebulização & 7 & 0,2 \\
Pegar medicamento & 857 & 19,1 \\
Realização de exames & 196 & 4,4 \\
Vacina & 632 & 14,1 \\
Outros & 262 & 5,9 \\
Total & 4.476 & 100,0
\end{tabular}

Recebeu visita domiciliar de um profissional da UBS no último ano?

\begin{tabular}{lrr} 
Não & 2.903 & 64,9 \\
Sim & 1.091 & 24,4 \\
Não sabe, ou não quis responder & 482 & 10,8 \\
Total & 4.476 & 100,0 \\
\hline
\end{tabular}


caracterização geral do atendimento no serviço de saúde dos usuários incluídos no estudo.

Quando avaliado o grau de Satisfação Geral dos usuários em relação ao serviço ofertado, a maioria demonstrou estar satisfeita, com 54,9\% optando pelo item "Satisfeito" e 23,0\% "Muito Satisfeito". Quando questionados em relação à Chance de Recomendar o serviço para outras pessoas, prevaleceram as opções "Alta" $(43,4 \%)$ e "Muito Alta" (17,7\%). A Figura 1 apresenta os dados detalhados sobre o nível de satisfação dos usuários e as chances de os mesmos recomendarem os serviços oferecidos pelas Unidades Básicas de Saúde.

Foram realizadas análises de associação entre as variáveis de desfecho: "Nível Geral de Satisfação dos usuários” e "Chance do Usuário Recomendar a UBS" com as variáveis sociodemográficas e o tipo de atendimento no serviço de saúde. As variáveis que tiveram nível de significância $\geq$ 90\% foram incluídas em um modelo de Regressão Logística Ordinal. O teste de desvio de adequação do ajuste indicou que o modelo para "Nível Geral de Satisfação" teve um bom ajuste para os dados observados, $\chi^{2}=7287,883, p=0,700$. A Tabela 2 apresenta os resultados desta Regressão e demonstrou que:

- As chances de mulheres estarem menos satisfeitas foi de 1,2 vezes a dos homens. ( $\mathrm{p}=$ $0,010)$.
- Usuários de cor/etnia branca têm 1,3 vezes mais chances de estarem mais satisfeitos do que os de cor/etnia preta. $(\mathrm{p}=0,021)$.

- As chances de usuários que não foram atendidos estarem menos satisfeito foi de 12,1 vezes a dos que foram atendidos. ( $\mathrm{p}<0,0005)$.

- Quanto ao motivo principal da visita a UBS, tendem a estar menos satisfeitos os que foram para agendar consulta $(1,4$ vezes, $\mathrm{p}=0,003)$, consultas sem agendamento prévio (1,2 vezes, $\mathrm{p}=0,032)$, pegar medicamentos $(1,7$ vezes, $\mathrm{p}<$ $0,0005)$ e tomar vacina $(1,7$ vezes, $\mathrm{p}<0,0005)$ em relação aos que foram com consulta agendada.

- As chances de usuários que não receberam visitas domiciliar no último ano estarem menos satisfeitos foi de 1,7 vezes $(\mathrm{p}<0,0005)$ a dos que receberam visita domiciliar.

- As chances de os usuários estarem satisfeitos nos diferentes níveis de escolaridade é similar, bem como diferença entre a idade e a renda mensal familiar não influenciam na satisfação.

A mesma análise foi realizada para determinar quais variáveis da caracterização sociodemográfica e caracterização geral do atendimento no serviço de saúde dos usuários têm um efeito na variável "Chance do Usuário Recomendar a UBS” (Tabela 3). O teste de desvio de adequação do ajuste indicou que o modelo para "Chance de Recomendar a UBS" teve um bom ajuste para os dados observados, $\chi 2=9110,794887, \mathrm{p}=0,659$.

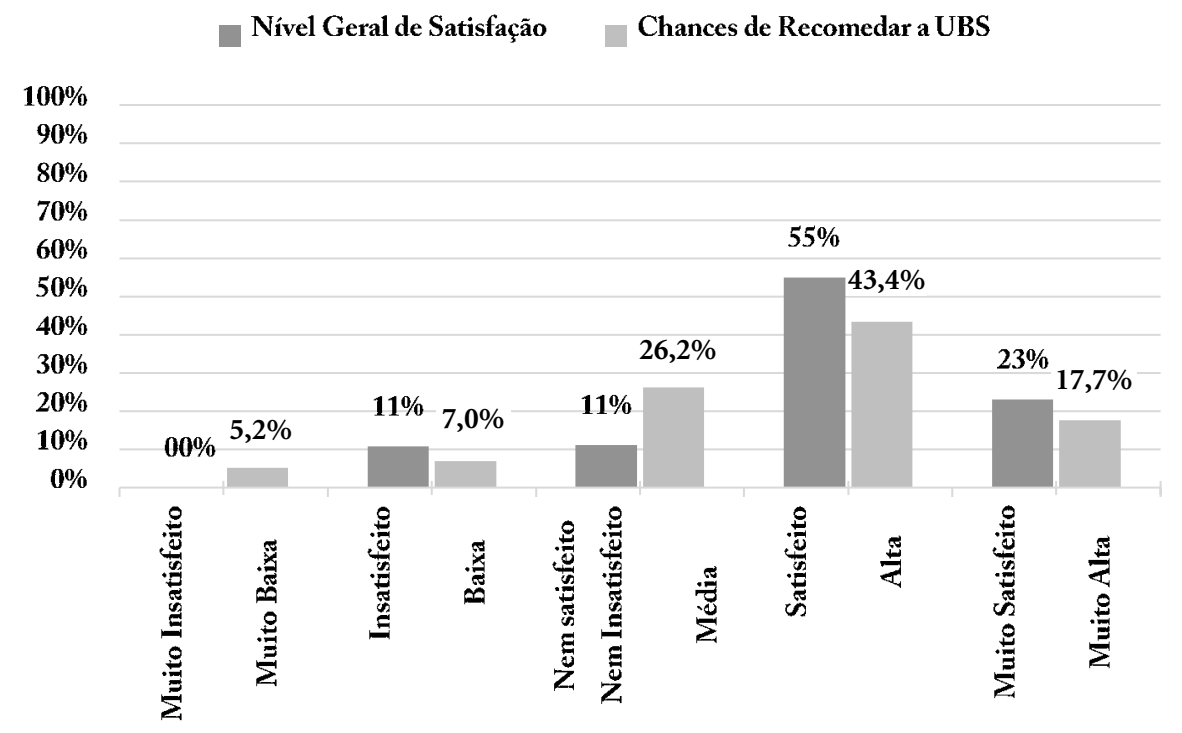

Figura 1. Caracterização do Nível Geral de Satisfação dos usuários e Chances do Usuário Recomendar a Unidade Básica de Saúde para amigos, conhecidos ou familiares. Distrito Federal, 2018-2019. 
Tabela 2. Fatores determinantes do Nível Geral de Satisfação dos usuários quanto ao atendimento nas Unidades Básicas de Saúde. Distrito Federal 2018-2019.

\begin{tabular}{|c|c|c|c|c|c|}
\hline \multirow{2}{*}{ Parâmetro } & \multirow{2}{*}{$\begin{array}{c}\operatorname{Exp}(B) \\
\text { Odds Ratio }\end{array}$} & \multicolumn{2}{|c|}{$\begin{array}{c}\text { 95\% Intervalo de Confiança } \\
\text { de Wald para } \operatorname{Exp}(B)\end{array}$} & \multicolumn{2}{|c|}{ Teste de hipótese } \\
\hline & & Inferior & Superior & Qui $^{2}$ de Wald & p valor \\
\hline Mulher & 1,227 & 1,049 & 1,436 & 6,553 & 0,010 \\
\hline Homem & 1 & . & . & . & . \\
\hline Amarela & 0,931 & 0,643 & 1,348 & 0,144 & 0,704 \\
\hline Branca & 0,776 & 0,625 & 0,962 & 5,348 & 0,021 \\
\hline Indígena & 1,268 & 0,608 & 2,645 & 0,401 & 0,526 \\
\hline Parda & 0,877 & 0,740 & 1,040 & 2,269 & 0,132 \\
\hline Preta & 1 & . & . & . & . \\
\hline Sem Estudo & 0,983 & 0,682 & 1,418 & 0,008 & 0,928 \\
\hline Ensino Fundamental & 0,909 & 0,710 & 1,163 & 0,578 & 0,447 \\
\hline Ensino Médio & 0,988 & 0,790 & 1,235 & 0,012 & 0,913 \\
\hline Ensino Superior & 1 & . & . & . & . \\
\hline Não Foi Atendido & 12,069 & 10,036 & 14,513 & 700,736 & $<0,001$ \\
\hline Foi Atendido & 1 & . & . & . & . \\
\hline Agendamento de consulta & 1,418 & 1,125 & 1,788 & 8,745 & 0,003 \\
\hline Consulta sem agendamento prévio & 1,247 & 1,019 & 1,525 & 4,600 & 0,032 \\
\hline Curativo & 0,647 & 0,410 & 1,021 & 3,495 & 0,062 \\
\hline Grupo Educativo & 1,650 & 0,774 & 3,519 & 1,680 & 0,195 \\
\hline Nebulização & 0,466 & 0,096 & 2,273 & 0,892 & 0,345 \\
\hline Pegar medicamento & 1,685 & 1,375 & 2,066 & 25,312 & $<0,001$ \\
\hline Realização de exames & 1,089 & 0,771 & 1,537 & 0,233 & 0,630 \\
\hline Vacina & 1,715 & 1,375 & 2,138 & 22,980 & $<0,001$ \\
\hline Consulta agendada & 1 & . & . & . & \\
\hline Não Recebeu Visita Domiciliar & 1,701 & 1,462 & 1,979 & 47,386 & $<0,001$ \\
\hline Não Lembra se Recebeu Visita & 1,484 & 1,172 & 1,878 & 10,740 & 0,001 \\
\hline Recebeu Visita Domiciliar & 1 & . & . & . & \\
\hline Idade (média) & 0,998 & 0,993 & 1,003 & 0,413 & 0,520 \\
\hline Renda Mensal (média) & 1,000 & 1,000 & 1,000 & 2,725 & 0,099 \\
\hline
\end{tabular}

Os resultados da Regressão Logística Ordinal para a Chance do Usuário Recomendar a UBS indicaram que:

- Não há diferença entre mulheres e homens quanto à Chance do Usuário Recomendar a UBS. Assim como para cor/etnia, escolaridade, idade e renda mensal familiar.

- As chances de usuários que não foram atendidos recomendar a UBS foram 5,1 vezes menores comparado aos que foram atendidos $(\mathrm{p}<$ $0,0005)$.

- Quanto ao motivo principal da visita à UBS, tende a diminuir a chance de recomendar a UBS os que foram para agendar consulta $(\mathrm{OR}=1,3$ $\mathrm{p}=0,020)$, pegar medicamentos $(\mathrm{OR}=1,3, \mathrm{p}=$ $0,003)$ e tomar vacina $(\mathrm{OR}=1,7 \mathrm{p}<0,0005)$ em relação aos que foram com consulta agendada.

- As chances de usuários que não receberam visitas domiciliar no último ano tenderem a não recomendar a UBS foi de 1,5 vezes $(\mathrm{p}<0,0005)$ a dos que receberam visita domiciliar.

O usuário ter ou não obtido o atendimento que buscava no dia da entrevista foi o fator que mais impactou tanto na satisfação (OR 12,1) quanto na chance de recomendar a UBS (OR 5,1).

Para verificar se há alguma correlação entre ser ou não atendido e outras variáveis, foi aplicado o teste de Qui-quadrado para independência, apresentado na Tabela 4.

Houve correlação estatisticamente significativa entre "Ser ou não Atendido" e "Sexo" $(\chi 2=4,19$ p = 0,041); "Cor/Etnia" $(\chi 2=13,45$ $\mathrm{p}=0,009)$, "Visita Domiciliar" ( $\chi 2=30,63 \mathrm{p}<$ $0,0005)$, porém todas foram associações muito fracas. Não houve correlação estatisticamente significante com "Escolaridade", "Faixa Etária" e "Renda Familiar". 
Tabela 3. Fatores determinantes das Chances do Usuário Recomendar as Unidades Básicas de Saúde para amigos, conhecidos ou familiares. Distrito Federal 2018-2019.

\begin{tabular}{|c|c|c|c|c|c|}
\hline \multirow[t]{2}{*}{ Parâmetro } & \multirow{2}{*}{$\begin{array}{c}\operatorname{Exp}(B) \\
\text { Odds Ratio }\end{array}$} & \multicolumn{2}{|c|}{$\begin{array}{c}\text { 95\% Intervalo de Confiança } \\
\text { de Wald para } \operatorname{Exp}(B)\end{array}$} & \multicolumn{2}{|c|}{ Teste de hipótese } \\
\hline & & Inferior & Superior & Qui $^{2}$ de Wald & p valor \\
\hline Mulher & 1,025 & 0,883 & 1,190 & 0,102 & 0,749 \\
\hline Homem & 1 & . & . & . & . \\
\hline Amarela & 1,256 & 0,885 & 1,784 & 1,629 & 0,202 \\
\hline Branca & 1,050 & 0,855 & 1,289 & 0,214 & 0,643 \\
\hline Indígena & 1,759 & 0,879 & 3,518 & 2,548 & 0,110 \\
\hline Parda & 1,100 & 0,936 & 1,293 & 1,327 & 0,249 \\
\hline Preta & 1 & . & . & . & . \\
\hline Sem Estudo & 1,225 & 0,866 & 1,733 & 1,313 & 0,252 \\
\hline Ensino Fundamental & 0,920 & 0,728 & 1,163 & 0,484 & 0,486 \\
\hline Ensino Médio & 1,001 & 0,809 & 1,238 & 0,000 & 0,994 \\
\hline Ensino Superior & 1 & . & . & . & . \\
\hline Não Foi Atendido & 5,104 & 4,314 & 6,040 & 360,625 & $<0,0005$ \\
\hline Foi Atendido & 1 & . & . & . & . \\
\hline Agendamento de consulta & 1,298 & 1,042 & 1,617 & 5,412 & 0,020 \\
\hline Consulta sem agendamento prévio & 1,069 & 0,883 & 1,295 & 0,467 & 0,494 \\
\hline Curativo & 0,800 & 0,517 & 1,239 & 1,000 & 0,317 \\
\hline Grupo Educativo & 0,845 & 0,413 & 1,732 & 0,210 & 0,647 \\
\hline Nebulização & 0,248 & 0,051 & 1,200 & 3,004 & 0,083 \\
\hline Pegar medicamento & 1,344 & 1,109 & 1,628 & 9,113 & 0,003 \\
\hline Realização de exames & 1,004 & 0,724 & 1,394 & 0,001 & 0,980 \\
\hline Vacina & 1,680 & 1,364 & 2,068 & 23,896 & $<0,0005$ \\
\hline Consulta agendada & 1 & . & . & . & . \\
\hline Não Recebeu Visita Domiciliar & 1,498 & 1,297 & 1,731 & 30,132 & $<0,0005$ \\
\hline Não Lembra se Recebeu Visita & 1,565 & 1,250 & 1,960 & 15,287 & $<0,0005$ \\
\hline Recebeu Visita Domiciliar & 1 & . & . & . & . \\
\hline Idade (média) & 0,998 & 0,994 & 1,003 & 0,509 & 0,476 \\
\hline Renda Mensal (média) & 1,000 & 1,000 & 1,000 & 0,524 & 0,469 \\
\hline
\end{tabular}

\section{Discussão}

Os resultados da pesquisa poderão contribuir para que gestores públicos organizem os serviços de saúde a partir das informações apresentadas na avaliação dos usuários. Os achados, apesar de serem locais e situados na região Leste de Saúde do Distrito Federal, são semelhantes à realidade de grandes centros urbanos, que cotidianamente se deparam com o desafio de identificar os fatores que favoreçam o acesso e a vinculação dos usuários aos serviços.

Além disso, os resultados mostraram que, apesar das dificuldades vivenciadas no Brasil para a implantação dos serviços na APS, as unidades avaliadas no DF têm conseguido responder positivamente, na percepção dos usuários, aos principais motivos que os levaram a comparecer na unidade - para a consulta agendada, a retirada de medicamento e para a consulta sem agendamento prévio. A pesquisa revelou que estes importantes dispositivos organizativos investigados, como a oferta de consulta, dispensação de medicamentos e visitas domiciliares podem influenciar na relação de vínculo e adesão ao serviço, uma vez que, tendo sua demanda atendida - como verificado na correlação da satisfação ao motivo da ida ao serviço -, os usuários tendem a estabelecer um bom vínculo de permanência, possibilitando a longitudinalidade do cuidado, e inclusive, recomendam a UBS a outras pessoas. Dessa maneira, quando a nova PNAB de 2017 propõe mudanças que fragilizam dispositivos como a atuação do ACS, a relativização da cobertura e a recomposição das equipes, colocam em risco a continuidade das ações e programas que visam ampliar 
Tabela 4. Correlação entre o usuário ter ou não ter obtido o atendimento que buscava no dia da entrevista e variáveis sociodemográficas. Distrito Federal, 2018-2019.

\begin{tabular}{|c|c|c|c|c|c|c|c|c|c|c|c|}
\hline \multirow{2}{*}{\multicolumn{2}{|c|}{ Categorias }} & & \multicolumn{6}{|c|}{ Recebeu Atendimento } & \multirow{2}{*}{$\begin{array}{c}\text { Qui }^{2} \text { de } \\
\text { Pearson }^{\star}\end{array}$} & \multirow{2}{*}{ Significância ${ }^{\star *}$} & \multirow{2}{*}{$\begin{array}{c}\text { V de } \\
\text { Cramer }^{\star * *}\end{array}$} \\
\hline & & & \multicolumn{2}{|c|}{ Não } & \multicolumn{2}{|c|}{ Sim } & \multicolumn{2}{|c|}{ Total } & & & \\
\hline \multirow{4}{*}{ 离 } & Mulher & $\mathrm{N}(\%)$ & 656 & $(19,0)$ & 2801 & $(81,0)$ & 3457 & $(100,0)$ & 4,19 & 0,041 & 0,031 \\
\hline & & RA & 2 & & -2 & & & & & & \\
\hline & Homem & N (\%) & 162 & $(16,1)$ & 842 & $(83,9)$ & 1004 & $(100,0)$ & & & \\
\hline & & RA & -2 & & 2 & & & & & & \\
\hline \multirow{10}{*}{ 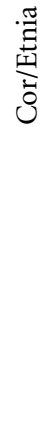 } & Amarela & $\mathrm{N}(\%)$ & 28 & $(17,2)$ & 135 & $(82,8)$ & 163 & $(100,0)$ & 13,45 & 0,009 & 0,055 \\
\hline & & RA & $-0,4$ & & 0,4 & & & & & & \\
\hline & Branca & $\mathrm{N}(\%)$ & 144 & $(19,1)$ & 609 & $(80,9)$ & 753 & $(100,0)$ & & & \\
\hline & & RA & 0,6 & & $-0,6$ & & & & & & \\
\hline & Indígena & $\mathrm{N}(\%)$ & 13 & $(37,1)$ & 22 & $(62,9)$ & 35 & $(100,0)$ & & & \\
\hline & & RA & 2,9 & & $-2,9$ & & & & & & \\
\hline & Parda & N (\%) & 462 & $(17,3)$ & 2214 & $(82,7)$ & 2676 & $(100,0)$ & & & \\
\hline & & RA & $-2,3$ & & 2,3 & & & & & & \\
\hline & Preta & N (\%) & 172 & $(20,5)$ & 666 & $(79,5)$ & 838 & $(100,0)$ & & & \\
\hline & & RA & 1,8 & & $-1,8$ & & & & & & \\
\hline \multirow{8}{*}{ 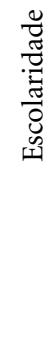 } & Não Estudou & N (\%) & 35 & $(14,1)$ & 214 & $(85,9)$ & 249 & $(100,0)$ & 5,95 & 0,114 & 0,037 \\
\hline & & $\mathrm{RA}$ & $-1,8$ & & 1,8 & & & & & & \\
\hline & Ensino & N (\%) & 310 & $(19,7)$ & 1266 & $(80,3)$ & 1576 & $(100,0)$ & & & \\
\hline & Fundamental & $\mathrm{RA}$ & 1,7 & & $-1,7$ & & & & & & \\
\hline & Ensino Médio & N (\%) & 384 & $(17,6)$ & 1792 & $(82,4)$ & 2176 & $(100,0)$ & & & \\
\hline & & RA & $-1,2$ & & 1,2 & & & & & & \\
\hline & Ensino Superior & N (\%) & 90 & $(19,4)$ & 374 & $(80,6)$ & 464 & $(100,0)$ & & & \\
\hline & & RA & 0,6 & & $-0,6$ & & & & & & \\
\hline \multirow{6}{*}{ 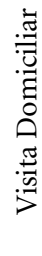 } & Não & N (\%) & 548 & $(18,9)$ & 2348 & $(81,1)$ & 2896 & $(100,0)$ & 30,63 & 0,000 & 0,083 \\
\hline & & RA & 1,4 & & $-1,4$ & & & & & & \\
\hline & Não Sabe & $\mathrm{N}(\%)$ & 121 & $(25,2)$ & 360 & $(74,8)$ & 481 & $(100,0)$ & & & \\
\hline & & RA & 4,1 & & $-4,1$ & & & & & & \\
\hline & Sim & N (\%) & 150 & $(13,8)$ & 938 & $(86,2)$ & 1088 & $(100,0)$ & & & \\
\hline & & RA & $-4,5$ & & 4,5 & & & & & & \\
\hline \multirow{8}{*}{ 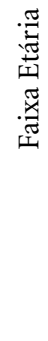 } & Jovem & N (\%) & 159 & $(17,7)$ & 737 & $(82,3)$ & 896 & $(100,0)$ & 1,42 & 0,701 & 0,018 \\
\hline & & RA & $-0,5$ & & 0,5 & & & & & & \\
\hline & Adulto & N (\%) & 377 & $(19,1)$ & 1596 & $(80,9)$ & 1973 & $(100,0)$ & & & \\
\hline & & RA & 1,2 & & $-1,2$ & & & & & & \\
\hline & Meia Idade & N (\%) & 166 & $(17,6)$ & 778 & $(82,4)$ & 944 & $(100,0)$ & & & \\
\hline & & RA & $-0,7$ & & 0,7 & & & & & & \\
\hline & Idoso & N (\%) & 117 & $(17,9)$ & 535 & $(82,1)$ & 652 & $(100,0)$ & & & \\
\hline & & RA & $-0,3$ & & 0,3 & & & & & & \\
\hline \multirow{10}{*}{ 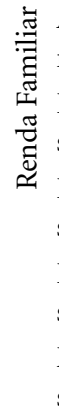 } & Até 01 salário & N (\%) & 182 & $(18,7)$ & 789 & $(81,3)$ & 971 & $(100,0)$ & 0,85 & 0,931 & 0,015 \\
\hline & mínimo & RA & 0,3 & & $-0,3$ & & & & & & \\
\hline & De 01 a 03 & $\mathrm{~N}(\%)$ & 394 & $(18,2)$ & 1766 & $(81,8)$ & 2160 & $(100,0)$ & & & \\
\hline & salários mínimos & RA & $-0,3$ & & 0,3 & & & & & & \\
\hline & De 03 a 05 & $\mathrm{~N}(\%)$ & 64 & $(17,7)$ & 298 & $(82,3)$ & 362 & $(100,0)$ & & & \\
\hline & salários mínimos & RA & $-0,4$ & & 0,4 & & & & & & \\
\hline & De 05 a 15 & $\mathrm{~N}(\%)$ & 24 & $(21,1)$ & 90 & $(78,9)$ & 114 & $(100,0)$ & & & \\
\hline & salários mínimos & RA & 0,7 & & $-0,7$ & & & & & & \\
\hline & Mais que 15 & $\mathrm{~N}(\%)$ & 1 & $(14,3)$ & 6 & $(85,7)$ & 7 & $(100,0)$ & & & \\
\hline & salários mínimos & RA & $-0,3$ & & 0,3 & & & & & & \\
\hline
\end{tabular}


e qualificar a cobertura e o acesso da primeira porta de entrada da população ao SUS.

Nesse sentido, ausência da visita do ACS, por exemplo, pode interferir na vinculação e satisfação dos usuários com os serviços, uma vez que os resultados mostraram que usuários que não receberam visitas domiciliar no último ano tinham menos chance de recomendar a UBS. Por isso, a visitação dos ACS na comunidade é fundamental para a longitudinalidade do atendimento, vínculo com o serviço e efetividade da $\mathrm{ESF}^{17-19}$.

A Atenção Primária à Saúde no Brasil tem sofrido desmontes estruturais que impactam principalmente as populações mais vulneráveis, como notado na nova apresentação da PNAB de 2017, que tem contribuído para fragilizar importantes conquistas que fortalecem a APS. A austeridade fiscal que tem sido imposta no Brasil, com mais rigor a partir de 2016, também tem contribuído para a redução crescente da oferta das tecnologias de cuidados e, já colocam em risco o mínimo de serviços públicos ofertados à população. $\mathrm{A}$ aprovação da Emenda Constitucional 95 (EC 95) em dezembro de 2016, que congela investimentos públicos na área social por 20 anos, traz consequências desastrosas à população, especialmente os mais pobres, pois afeta a organização e oferta dos serviços de saúde públicos e os equipamentos da assistência social, que estão voltados para o enfrentamento das desigualdades sociais. Deste modo, estudos como o presente, que demostram quais estratégias fomentam a vinculação da comunidade aos serviços públicos de saúde, são estruturantes para momentos de cortes e ameaças à continuidade da implementação do SUS, como o que está em vigência. Ao identificarmos, pelas pesquisas, os fatores que aproximam com satisfação a comunidade dos serviços, mapeamos pontos de atenção que a população reconhece como a vertente positiva do cuidado, e pudemos assim, nos apropriar destas informações para planejar ações que conscientizem a população que esses cuidados estão em risco de serem extintos, e da necessidade de uma participação mais ativa da comunidade na gestão e controle social da saúde.

Os usuários entrevistados revelaram que dentre os principais motivos de terem buscado os serviços, estão o acesso ao Programa Nacional de Imunizações e a retirada de medicamentos na farmácia da Unidade. Dos entrevistados, 14,1\% responderam que compareceram na unidade no dia da entrevista para vacinação e $19,1 \%$ buscaram a farmácia. Estas portas de acesso que possibilitam a longitudinalidade do cuidado, a integralidade e atenção ao primeiro contato, estão entre as tecnologias de cuidados que já sofrem impacto com o subfinanciamento do SUS. O fechamento ou a redução destes acessos poderão inserir no mapa de saúde, o retorno de doenças já erradicadas e o agravamento dos problemas de saúde, principalmente dos problemas mentais, que em tempos de crise econômica, recessão e consequente perda do emprego se manifestam em quadros de depressão, ansiedade e violência autoprovocada que geralmente demandam medicamentos de alto custo.

Atualmente na região leste de saúde do DF, como mostraram os dados da pesquisa, os usuários que acessam a consultas, tanto agendadas, como encaixadas, avaliaram em sua prevalência, como satisfatórias, o que indica que a população assistida parece ter respondido sua demanda para avaliação de seu estado de saúde nos serviços da APS. Novos estudos precisam ser incorporados na rotina de avaliação das Redes de Atenção à Saúde para compreender se esta população está sendo assistida nos diversos níveis de sofisticação tecnológica e o grau de satisfação com as práticas de saúde. Compreender qual o grau de satisfação dos sujeitos no contínuo do cuidado, na longitudinalidade, desde a APS até níveis mais complexos do sistema, nos apoia também a compreender a noção de como a integralidade da atenção à saúde tem sido construída em rede.

Para que os processos de avaliação fomentem também a garantia da implementação da integralidade nos serviços, partimos da compreensão do que foi sistematizado como os três conjuntos de sentidos sobre a 'integralidade' - como traço da boa medicina; como modo de organizar as práticas e como respostas governamentais a problemas específicos de saúde ${ }^{20}$. No que se refere à integralidade como traço da boa medicina, identificamos que quando conseguimos acessar na presente pesquisa, a percepção dos usuários se os serviços atendem as necessidades e expectativas da população, estamos nos aproximando de uma possível compreensão do que seria a "boa medicina" discutida pelos autores. Considerando que $24 \%$ das pessoas que responderam a pesquisa informaram que compareceram na unidade para consulta agendada e que o grau de satisfação geral dos usuários em relação ao serviço esteve entre "satisfeito" (54,9\%) e "muito satisfeito" $(23,0 \%)$, podemos inferir que apesar da positividade na percepção, é necessário que as equipes analisem em seus processos de cuidado como tem sido organizado o modelo de atenção, e se os usuários que estão sendo acolhidos têm tido a escuta de suas necessidades de forma desfragmen- 
tada e compromissada com um projeto de defesa da vida, como se propõe o SUS, principalmente na APS.

\section{Conclusão}

Destacamos que a experiência desta pesquisa ratifica que a avaliação localizada pode ser um potente dispositivo para superar as dificuldades que ainda perduram para oferecer os arranjos locais dos serviços de saúde que contemple a atenção integral. A abertura da escuta qualificada de modo que usuários pudessem avaliar os componentes da organização das práticas para viabilizar o acesso e qualidade nos serviços, despontam como novos canais participativos, que em tempos de ameaças e desmontes dos serviços de saúde, se apresentam como alternativas para

\section{Colaboradores}

DLC Furlanetto participou do delineamento do estudo, análise e interpretação dos dados, redação e revisão do manuscrito. AA Lima trabalhou na concepção e delineamento, análise e interpretação dos dados, e redação do artigo. CM Pedrosa participou da concepção, delineamento e redação do artigo. TTB Paranaguá participou na concepção e delineamento do estudo, e redação do artigo. MF Xavier participou do delineamento do estudo, coleta dos dados, e redação do artigo. AKP Silva participou da coleta de dados e redação do manuscrito. VB Rodrigues participou do delineamento do estudo e redação do artigo. LMP Santos participou do delineamento, obteve financiamento e coordenou o estudo; supervisionou a coleta de dados em campo, participou da análise e interpretação dos dados, e da redação do manuscrito. Todos os autores aprovaram a versão final do manuscrito. que a população possa manifestar sua posição em relação às respostas governamentais oferecidas a problemas específicos de saúde.

Concluiu-se que para a maioria dos usuários entrevistados neste estudo a percepção é que os serviços são satisfatórios. Recomenda-se aos gestores locais providências para reduzir a ocorrência de casos que, apesar de ter tido acesso à Unidade Básica de Saúde, não conseguiram o atendimento pretendido no dia da pesquisa, o que irá repercutir favoravelmente no nível de satisfação. O estudo também trouxe evidências que corroboram a importância de os serviços de Atenção Primária contarem com Equipes de Saúde da Família completas, inclusive com Agentes Comunitários de Saúde, que possam efetivamente expandir as visitas domiciliares e deste modo promover e estreitar os vínculos com os usuários.

\section{Agradecimentos}

Os autores agradecem à população da Região Leste do Distrito Federal pela disponibilidade e colaboração para o desenvolvimento desse estudo.

\section{Apoio Financeiro}

O estudo foi contemplado na Chamada Pública FAPDF/MS-DECIT/CNPQ/SESDF No 001/2016, financiada pela FAP/DF e Departamento de Ciência e Tecnologia do Ministério da Saúde. 


\section{Referências}

1. Contandriopoulos A, Champagne F, Denis J, Pineault R. Avaliação na área da saúde: conceitos e métodos. In: Hartz ZA, organizador. Avaliação em saúde: dos modelos conceituais à prática na análise da implantação de programas. Rio de Janeiro: Fiocruz; 1997. p. 29-47.

2. Gaioso VP, Mishina SM. User satisfaction from the perspective of acceptability in the family health scenario. Texto Contexto Enferm 2007; 16(4):617-625.

3. Serapioni M, Romaní O. Potencialidades e desafios da participação em instâncias colegiadas dos sistemas de saúde: os casos de Itália, Inglaterra e Brasil. Cad Saude Publica 2006; 22(11):2411-2421.

4. Almeida CAL, Tanaka OY. Meta-avaliação de um processo participativo no fortalecimento da gestão municipal. Rev Saude Publica 2017; 51(99):1-9.

5. Arruda CAM, Bosi MLM. User's satisfaction of primary health care: a qualitative study in the Northeast of Brazil. Interface (Botucatu) 2017; 21(61):321-332.

6. Esperidião MA, Trad LAB. Avaliação de satisfação de usuários. Cien Saude Colet 2005; 10(Supl.):303-312.

7. Esperidião MA, Trad LAB. Avaliação de satisfação de usuários: considerações teórico-conceituais. Cad Saude Publica 2006; 22(6):1267-1276.

8. Institute of Medicine (US), Committee on Quality of Health Care in America. Crossing the quality chasm: a new health system for the 21st century. Washington: National Academy Press; 2001.

9. Reino Unido. Health and Social Care Act 2012. Section 23, $13 H$ [página na Internet]. Disponível em: http:// www.legislation.gov.uk/ukpga/2012/7/section/23

10. Brasil. Ministério da Saúde (MS). Secretaria de Atenção à Saúde. Política Nacional de Humanização. Documento Base. $4^{\mathrm{a}}$ ed. Brasília: MS; 2007.

11. Silva RR, Brandão DB. Nas rodas da avaliação participativa. In: Campos OR, Furtado JP. Desafios da avaliação de programas e serviços em saúde. Campinas: UNICAMP; 2011. p. 59-84.

12. Comes Y, Trindade JS, Shimizu HE, Hamann EM, Bargioni F, Ramirez L, Sanchez MN, Santos LMP. Avaliação da satisfação dos usuários e da responsividade dos serviços em municípios inscritos no Programa Mais Médicos. Cien Saude Colet 2016; 21(9):2749-2759.

13. Starfield B. Atención primaria: Equilibrio entre necesidades de salud, servicios y tecnologia. Barcelona: Masson; 2004.
14. Hollanda E, Siqueira SAV, Andrade GRB, Molinaro A, Vaitsman J. Satisfação e responsividade em serviços de atenção à saúde da Fundação Oswaldo Cruz. Cien Saude Colet 2012; 17(12):3343-3352.

15. Governo do Distrito Federal. Secretaria de Planejamento, Orçamento e Gestão. Companhia de Planejamento do Distrito Federal (CODEPLAN). Pesquisa Distrital por Amostra de Domicílios do Distrito Federal - PDAD-DF - 2015 [documento na Internet]. Brasília: CODEPLAN; 2016. Disponível em: http://www.codeplan.df.gov.br/wp-content/uploads/2018/02/PDADDistrito-Federal-1.pdf

16. Pasquali L. Instrumentação psicológica: fundamentos e práticas. Dados eletrônicos. Porto Alegre: Artmed; 2010.

17. Almeida MLB. Vulnerabilidade familiar: concepções dos agentes comunitários de saúde. [dissertação]. Rio de Janeiro: Fiocruz/ENSP; 2015.

18. Bezerra YRN, Feitosa MZS. A afetividade do agente comunitário de saúde no território: um estudo com os mapas afetivos. Cien Saude Colet 2018; 23(3):813822.

19. Costa SM, Araújo FF, Martins LV, Nobre LLR, Araújo FM, Rodrigues CAQ. Agente Comunitário de Saúde: elemento nuclear das ações em saúde. Cien Saude Colet 2013; 18(7):2147-2156.

20. Pinheiro R, Mattos RA, organizadores. Os sentidos da integralidade na atenção e no cuidado à saúde. Rio de Janeiro: CEPESC/IMS/UERJ/ABRASCO; 2005.
Artigo apresentado em 30/05/2019

Aprovado em 07/08/2019

Versão final apresentada em 14/11/2019 
